\title{
Effectiveness of antenatal corticosteroids in reducing respiratory disorders in late preterm infants: randomised clinical trial
}

\author{
Ana Maria Feitosa Porto, supervisor of medical residency programme in gynaecology and obstetrics, Isabela \\ Cristina Coutinho, preceptor of high risk ward, Jailson Barros Correia, research director, Melania Maria Ramos \\ Amorim, professor in postgraduate programme on maternal and child health
}

Instituto de Medicina Integral Professor Fernando Figueira (IMIP), Recife, Pernambuco, Brazil

Correspondence to: Melania Maria Ramos de Amorim, Rua Neuza Borborema de Souza, 300, Bairro Santo Antônio, 58406-120 Campina Grande, PB, Brazil melamorim@uol.com.br

Cite this as: BMJ 2011;342:d1696 doi:10.1136/bmj.d1696

\section{ABSTRACT}

Objectives To determine the effectiveness of corticosteroids in reducing respiratory disorders in infants born at 34-36 weeks' gestation.

Design Randomised triple blind clinical trial.

Setting A large tertiary teaching hospital in northeast of Brazil.

Participants Women at 34-36 weeks of pregnancy at risk of imminent premature delivery.

Interventions Betamethasone $12 \mathrm{mg}$ or placebo intramuscularly for two consecutive days.

Main outcomes measures Primary outcome was the incidence of respiratory disorders (respiratory distress syndrome and transient tachypnoea of the newborn). Secondary outcomes included the need for ventilatory support, neonatal morbidity, and duration of stay in hospital.

Results 320 women were randomised, 163 of whom were assigned to the treatment group and 157 to the controls. Final analysis included 143 and 130 infants, respectively. The rate of respiratory distress syndrome was low (two $(1.4 \%)$ in the corticosteroid group; one $(0.8 \%)$ in the placebo group; $\mathrm{P}=0.54)$, while the rate of transient tachypnoea was high in both groups (34 (24\%) v 29 $(22 \%) ; \mathrm{P}=0.77)$. There was no reduction in the risk of respiratory morbidity with corticosteroid use even after adjustment for subgroups of gestational age (34-34 ${ }^{+6}$ weeks, $35-35^{+6}$ weeks, and $\geq 36$ weeks). The adjusted risk of respiratory morbidity was 1.12 ( $95 \%$ confidence interval 0.74 to 1.70 ). The need for ventilatory support was around $20 \%$ in both groups. There was no difference in neonatal morbidity (88 (62\%) v $93(72 \%) ; \mathrm{P}=0.08)$ or in the duration of stay in hospital between the two groups (5.12 v 5.22 days; $\mathrm{P}=0.87$ ). Phototherapy for jaundice was required less often in babies whose mothers received corticosteroids (risk ratio $0.63,0.44$ to 0.91 ).

Conclusions Antenatal treatment with corticosteroids at 34-36 weeks of pregnancy does not reduce the incidence of respiratory disorders in newborn infants.

Trial registration Clinical Trials NCT00675246.

\section{INTRODUCTION}

Though late preterm infants were previously thought to be as physiologically and metabolically mature as term infants, we now know they are at a higher risk of morbidity and mortality. ${ }^{1-4}$ As late preterm infants represent the majority of premature infants, they have a considerable impact on the healthcare system compared with full term infants. ${ }^{5-8}$

Respiratory disorders are often associated with late prematurity and are more common in than in full term infants $\left(29 \% v 4 \%{ }^{3}\right)$. There is a greater risk of the infant requiring oxygen and ventilatory support and of being admitted to intensive care. ${ }^{910}$ The most common respiratory disorders are transient tachypnoea of the newborn, respiratory distress syndrome, pneumonia, and pulmonary hypertension. ${ }^{3}$

Prevention of these disorders has been a concern, and various investigators have suggested that antenatal treatment with corticosteroids could accelerate lung maturation in late preterm infants. ${ }^{11-13}$ Though the beneficial effects of corticosteroids before 34 weeks of pregnancy have been well established, ${ }^{14}{ }^{15}$ we do not know whether these effects extend beyond the 34th week.

Only limited evidence on this issue is available from two studies ${ }^{1617}$ included in the Cochrane review that addressed the outcomes of babies born at 33 and $34^{+6}$ weeks' gestation. ${ }^{15}$ The review concluded that there was a significant reduction in respiratory distress syndrome in babies born between 33 and $34^{+6}$ weeks' gestation but no significant reduction in babies born between 35 and $36^{+6}$ weeks' gestation (relative risk $0.61,95 \%$ confidence interval 0.11 to 3.26 , one study, 189 cases). ${ }^{15}$

We carried out a randomised controlled trial to determine the effectiveness of antenatal treatment with corticosteroids at 34-36 weeks of pregnancy in reducing the incidence of neonatal respiratory disorders. 
Table 1|Baseline characteristics of women at 34-36 weeks of pregnancy at risk of imminent premature delivery according to antenatal treatment with corticosteroids or placebo. Figures are numbers (percentages) unless stated otherwise

\begin{tabular}{|c|c|c|}
\hline Variable & Corticosteroid $(n=143)$ & Placebo $(n=130)$ \\
\hline Mean (SD) age (years) & $23.3(6.1)$ & $22.9(5.5)$ \\
\hline Median (IQR) No of pregnancies & $1(1-3)$ & $1.5(1-3)$ \\
\hline Primigravidas & $72(50)$ & $65(50)$ \\
\hline Median (IQR) No of deliveries & $0(0-1)$ & $0(0-1)$ \\
\hline Nulliparas & $78(55)$ & $69(53)$ \\
\hline \multicolumn{3}{|l|}{ Gestational age at admission (weeks): } \\
\hline Range & $34-36$ & $34-36$ \\
\hline Mean (SD) & $35.0(0.7)$ & $35.0(0.7)$ \\
\hline History of prematurity & $20(14)$ & $12(9)$ \\
\hline \multicolumn{3}{|l|}{ Associated conditions: } \\
\hline Hypertension & $33(23)$ & $36(28)$ \\
\hline Premature rupture of membranes & $54(38)$ & $54(42)$ \\
\hline Premature labour & $97(68)$ & $86(66)$ \\
\hline Diabetes & $3(2)$ & $2(2)$ \\
\hline Fetal growth restriction & $1(0.7)$ & $2(2)$ \\
\hline Oligohydramnios & $15(11)$ & $5(4)$ \\
\hline Others & $20(14)$ & $12(9)$ \\
\hline Received tocolytics (nifedipine) & $88(62)$ & $79(61)$ \\
\hline
\end{tabular}

\section{METHODS}

\section{Study design}

We carried out a randomised, triple blind, placebo controlled clinical trial between April 2008 and June 2010 at the Instituto de Medicina Integral Prof Fernando Figueira (IMIP), Recife, a large tertiary teaching hospital in the northeast of Brazil.

\section{Study population}

The sample size was calculated with open source software (Openepi version 2.3, Atlanta, GA), with an assumed $28.9 \%$ rate of respiratory disorders in late preterm infants, ${ }^{3}$ an $\alpha$ error of $5 \%$, and $80 \%$ power to detect a reduction of $50 \%$ in the rate of respiratory disorders with the use of corticosteroids. ${ }^{15}$ This resulted in a required sample size of 266 (133 in each group), which we increased to 320 to cater for losses and exclusions.

Pregnant women receiving care at the institute were included if they were at $34-36^{+6}$ weeks' gestation and were at risk of imminent premature delivery (either spontaneously or if early delivery was recommended as a result of problems with the mother or fetus, or both) at the time of admission to hospital. Gestational age was defined according to the date of the woman's last menstrual period, if known and reliable, or by ultrasonography before 20 weeks of pregnancy. Reasons for exclusion from the study included multiple pregnancy, major congenital malformations, haemorrhagic syndromes with active bleeding, clinical evidence of chorioamnionitis, previous use of corticosteroids, or need for immediate resolution of pregnancy for maternal or fetal reasons. Women who were discharged from hospital while still pregnant and who went on to deliver elsewhere were excluded from the study after randomisation.

\section{Randomisation and follow-up}

A statistician who was not involved in the study prepared a table of random numbers in a single block (random allocation software, version 1.0), with 163 women randomised to receive betamethasone and 157 to placebo. The hospital pharmacy prepared 320 sealed cardboard boxes, each containing four ampoules of betamethasone or placebo, identical in appearance, volume, and colour, and numbered in accordance with the table of random numbers. Each ampoule of betamethasone contained $6 \mathrm{mg}(3 \mathrm{mg}$ acetate and $3.9 \mathrm{mg}$ disodium phosphate) and was prepared by the Laboratório Mantecorp (Rio de Janeiro, Brazil). The placebo ampoules contained a similar volume of $0.9 \%$ saline solution and were prepared for this trial by the pharmacy department of the Clinics Hospital, University of San Paulo.

Only the pharmacist responsible for preparing the boxes was aware of their contents. The investigators, the physicians who cared for the women, the statistician, and the women themselves were unaware of the contents; this information was disclosed only after data analysis was complete.

The physicians in charge of the obstetrics department identified potentially eligible women, who were then approached by the investigators. Only after they had signed an informed consent form did those women agreeing to participate in the study receive the sealed cardboard box corresponding to their randomisation group. Two ampoules were applied intramuscularly, with two more being administered 24 hours later. The study investigators took no part in the prepartum or postpartum management of the women or in neonatal management. Women in premature labour underwent tocolysis with nifedipine, in accordance with the hospital routine practice, in an attempt to postpone delivery and allow the full course of medication to be administered.

The investigators and the neonatologist who followed up the infants prospectively collected data on the pregnant women and their newborns on a standardised form.

If the woman delivered before she received the second dose of the medication, she was analysed as part of the group to which she had been randomised (intention to treat analysis). If the woman completed the scheduled regimen but was discharged while still pregnant and went on to deliver in another hospital, she was considered a post-randomisation loss to follow-up.

\section{Outcomes evaluated}

The primary outcomes were the occurrence of neonatal respiratory disorders: respiratory distress syndrome or transient tachypnoea of the newborn, defined by the presence of respiratory distress for more than two hours after birth and characterised by tachypnoea, expiratory grunting, chest wall retractions, flaring of the nostrils, cyanosis, and a growing need for oxygen. ${ }^{3}$ We used radiological criteria to differentiate between the two disorders used: diffuse reticulogranular infiltrate in respiratory distress syndrome and thickening of the bronchovascular bundle, pulmonary 
Table 2 Perinatal outcomes in infants born at 34-36 weeks of pregnancy at risk of imminent premature delivery according to antenatal treatment with corticosteroids or placebo

\begin{tabular}{|c|c|c|c|c|}
\hline Variable & $\begin{array}{l}\text { Corticoster- } \\
\text { oid }(n=143)\end{array}$ & $\begin{array}{l}\text { Placebo } \\
(n=130)\end{array}$ & $\begin{array}{c}\text { Relative risk } \\
(95 \% \mathrm{Cl})\end{array}$ & $P$ value \\
\hline No (\%) of vaginal deliveries & $98(69)$ & $90(69)$ & $0.99(0.84$ to 1.16$)$ & 0.90 \\
\hline Mean (SD) gestation at delivery (weeks) & $35.6(1.17)$ & $35.5(1.08)$ & - & 0.71 \\
\hline No (\%) <37 weeks' gestation & 127 (89) & 119 (92) & 0.97 (0.90 to 1.05$)$ & 0.45 \\
\hline Mean (SD) birth weight (g) & $2640(445)$ & $2627(452)$ & - & 0.80 \\
\hline No $(\%)<2500 \mathrm{~g}$ & $49(34)$ & $51(39)$ & 0.87 (0.64 to 1.19$)$ & 0.40 \\
\hline No (\%) small for gestational age & $35(25)$ & $29(22)$ & $1.10(0.71$ to 1.69$)$ & 0.67 \\
\hline \multicolumn{5}{|l|}{ Apgar score: } \\
\hline Median (IQR) score at one minute & $8(8-9)$ & $8(8-9)$ & - & $0.20^{\star}$ \\
\hline No $(\%)<7$ at one minute & $19(13)$ & $19(15)$ & $0.91(0.50$ to 1.64$)$ & 0.75 \\
\hline Median (IQR) score at five minutes & $9(9-10)$ & $9(9-10)$ & - & $0.77^{\star}$ \\
\hline No (\%) $<7$ at five minutes & $1(1)$ & $2(2)$ & 0.45 (0.04 to 4.95$)$ & $0.46 \dagger$ \\
\hline
\end{tabular}

$\mathrm{IQR}=$ interquartile range.

*Mann-Whitney test.

†Fisher's exact test. needed to treat was calculated with its 95\% confidence interval for the outcomes in which we found a beneficial effect of corticosteroid treatment.

\section{RESULTS}

A total of 352 women fulfilled the admission criteria for the study. Of these, 320 agreed to participate and were randomised to receive betamethasone $(n=163)$ or placebo $(n=157)$. Forty three women were discharged from hospital during pregnancy and were lost to follow-up (19 and 24, respectively). Two other exclusions occurred after randomisation in the placebo group: one because a twin pregnancy was detected only after randomisation and one because the pregnancy was found to have already reached term. Therefore, 144 women remained in the corticosteroid group and 131 in the placebo group, with 13\% of losses after randomisation (43 cases). As one stillbirth occurred in each group, we analysed 143 babies in the intervention group and 130 in the control group (figure).

There were no significant differences between groups in most baseline characteristics (table 1). In both groups $78 \%$ of the women received the full regimen of two doses (111 in intervention group, 101 in placebo group), and there was no significant difference between the groups in this respect. The interval between administration of the last dose and delivery was a median of two days in both groups (interquartile range $1-4 ; \mathrm{P}=0.49)$. No local or systemic side effects occurred, and there were no unexpected effects or adverse reactions to corticosteroid treatment (data not shown in tables). Perinatal outcomes were similar (table 2).

In both groups there were low rates of respiratory distress syndrome (two cases $(1.4 \%)$ in the corticosteroid group and one case $(0.8 \%)$ in the placebo group; $\mathrm{P}=0.54)$ and high rates of transient tachypnoea of the newborn (34 cases $(24 \%)$ v 29 cases $(22 \%) ; \mathrm{P}=0.77$ ) (table 3). Treatment with corticosteroids failed to reduce the risk of any respiratory morbidity (risk ratio $1.09,95 \%$ confidence interval 0.72 to 1.66 ). Only one baby in the corticosteroid group required exogenous surfactant. Necessity for ventilatory support was also similar, at around $20 \%$ in both groups (28 cases $(20 \%)$ in the corticosteroid group and 24 cases (19\%) in the placebo group). There was no difference in the type of ventilatory support used, and the mean duration of ventilation was 2.2 days in the infants in the corticosteroid group and 2.8 days in the placebo group $(\mathrm{P}=0.65)$ (table 3$)$.

We performed a stratified analysis to detect any differences in the effect of corticosteroid by gestational age $(34,35$, and 36 weeks or more). The rate of respiratory disorders was 50\% (15/30), 25\% (28/111), and $17 \%$ (23/132), respectively, and there were no significant differences between the groups. The corticosteroid had no effect in reducing respiratory morbidity at 34,35 , and 36 or more weeks, and the risk of respiratory disorders for the use of corticosteroid adjusted for gestational age was 1.12 (0.74 to 1.70$)$ (table 4$)$. 
Table 3 | Respiratory complications in infants born to women at 34-36 weeks of pregnancy at risk of imminent premature delivery according to antenatal treatment with corticosteroids or placebo. Figures are numbers (percentages) unless stated otherwise

\begin{tabular}{|c|c|c|c|c|}
\hline Variable & $\begin{array}{l}\text { Corticoster- } \\
\text { oid }(n=143)\end{array}$ & $\begin{array}{l}\text { Placebo } \\
(n=130)\end{array}$ & $\begin{array}{l}\text { Risk ratio } \\
(95 \% \mathrm{Cl})\end{array}$ & $P$ value \\
\hline Respiratory morbidity & $36(25)$ & $30(23)$ & 1.09 (0.72 to 1.66$)$ & 0.69 \\
\hline \multicolumn{5}{|l|}{ Type of respiratory morbidity: } \\
\hline Respiratory distress syndrome & $2(1)$ & $1(1)$ & $1.82(0.17$ to 19.8$)$ & $0.54^{*}$ \\
\hline Transient tachypnoea & $34(24)$ & $29(22)$ & 1.07 (0.69 to 1.65$)$ & 0.77 \\
\hline Use of surfactant & $1(1)$ & 0 & $-\dagger$ & $0.52^{\star}$ \\
\hline \multicolumn{5}{|l|}{ Ventilatory support: } \\
\hline Any & $28(20)$ & 24 (19) & 1.06 (0.65 to 1.73$)$ & 0.81 \\
\hline Invasive (mechanical ventilation) & $2(1)$ & $1(1)$ & $1.82(0.17$ to 19.82$)$ & $0.54^{*}$ \\
\hline Non-invasive & $26(18)$ & $23(18)$ & 1.03 (0.62 to 1.71$)$ & 0.92 \\
\hline >1 day of ventilation & $10(36)$ & $11(46)$ & 0.78 (0.40 to 1.51$)$ & 0.46 \\
\hline Mean (SD) duration (days) & $2.2(2.8)$ & $2.8(5.9)$ & - & 0.65 \\
\hline
\end{tabular}

*Fisher's exact test.

†Not estimated.

Rates of jaundice (76 (53\%) v $73(57 \%))$, hypoglycaemia $(15(11 \%)$ v $9(7 \%))$, and sepsis $(6(4 \%)$ v $9(7 \%))$ were similar in the two groups. Nevertheless, the rate of jaundice requiring phototherapy was lower in babies whose mothers had received corticosteroids (34 $(24 \%)$ v $49(38 \%)$; $\mathrm{P}=0.01)$, resulting in a $47 \%$ lower risk of requiring phototherapy and a number needed to treat of 7 (4 to 33). There was no difference in the outcomes of neonatal morbidity (88 (61.5\%) v $93(71.5 \%))$, admission to intensive care $(47(33 \%) v 43(33 \%))$, or rate of neonatal death (two $(2 \%)$ in the placebo group). The

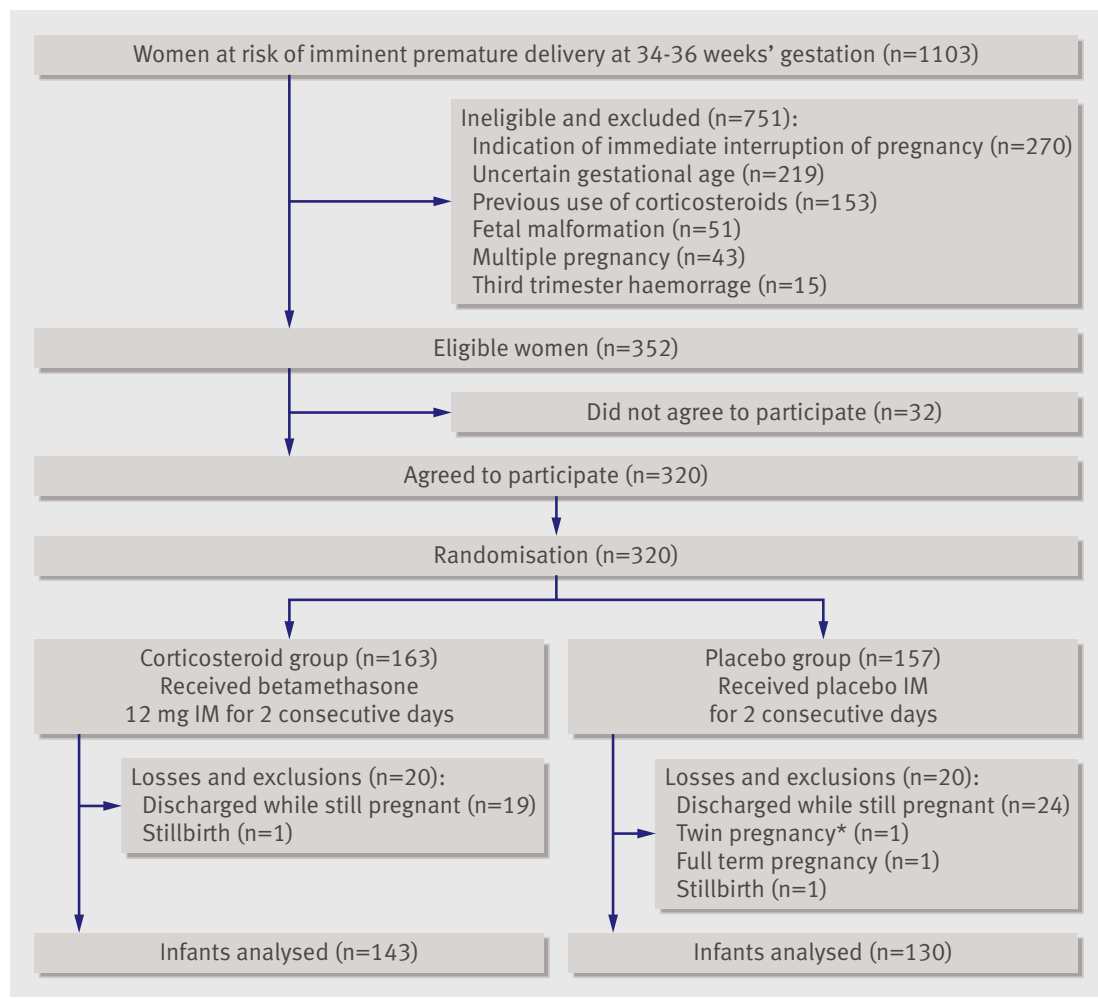

Procedures for selection and follow-up of participants. ${ }^{*}$ Confirmed only after randomisation. $\mathrm{IM}=$ intramuscular deaths in the placebo group were associated with severe perinatal asphyxia. The duration of stay in hospital was also similar in the two groups (5.1 v 5.2 days) (table 5).

\section{DISCUSSION}

Our randomised controlled trial shows that antenatal treatment with corticosteroids in women at 34-36 weeks of pregnancy at risk of imminent premature delivery is ineffective in reducing the occurrence of respiratory disorders in the babies and fails to exert any effect on the incidence of various other complications of late prematurity, with the exception of jaundice requiring phototherapy. Our study shows that late preterm infants develop high rates of transient tachypnoea (around 23\%) and neonatal morbidity, confirming observations reported over the past decade from a range of different settings. ${ }^{2-4}$

Antenatal treatment with corticosteroids has been used to accelerate lung maturation for more than three decades, and its effectiveness and safety is well established for pregnancies of up to 34 weeks. ${ }^{14-17} \mathrm{~A}$ possible extension of the benefits of corticosteroids beyond this gestational age, however, has only recently been more widely considered. ${ }^{11-13}$

\section{Comparison with other studies}

In two published meta-analyses, gestational age subgroups showed insufficient evidence to either recommend or discourage the use of corticosteroids after $34^{+6}$ weeks of pregnancy. ${ }^{1415}$ Although some studies have suggested a reduction in the risk of respiratory disorders, both the number of infants of this gestational age and the number of respiratory events were low, with insufficient power to detect any significant difference. In contrast, another meta-analysis published in 1995 showed that one would need to treat 94 pregnant women beyond 34 weeks' gestation to prevent one case of respiratory distress syndrome. ${ }^{19}$

The ASTECS study evaluated the effectiveness of antenatal corticosteroids in 998 women who were candidates for full term elective caesarean section and reported a significant reduction of around $54 \%$ in admissions to neonatal intensive care for respiratory disorders..$^{20}$ Nevertheless, that study had various methodological limitations: it was not blinded and there was no placebo group, the intervention group being compared with treatment referred to as "usual." In addition, despite a reduction in the rate of admission to neonatal intensive care, they found no reduction in the objective measures of respiratory morbidity, and, most importantly, their population of women with elective caesarean section before 40 weeks is different from the women in our study. The ASTECS study excluded women in labour and those with hypertensive syndromes and did not mention the state of the amniotic membranes, whereas in our study on late premature delivery, premature rupture of membranes and hypertensive syndromes were the most common conditions. 
Table 4 Stratified analysis: risk of respiratory morbidity according to gestational age and allocation to antenatal treatment with corticosteroids or placebo

\begin{tabular}{|c|c|c|c|}
\hline Variable & Respiratory morbidity & Risk ratio* $(95 \% \mathrm{Cl})$ & $P$ value \\
\hline \multicolumn{4}{|l|}{ All gestational ages } \\
\hline Corticosteroid group $(n=143)$ & $36(25)$ & $1.09(0.72 \text { to } 1.66)^{\star}$ & \multirow{2}{*}{0.69} \\
\hline Placebo group $(n=130)$ & $30(23)$ & 1.00 & \\
\hline \multicolumn{4}{|l|}{$34-34^{+6}$ weeks } \\
\hline Corticosteroid group $(n=13)$ & $7(54)$ & 1.14 (0.56 to 2.33$)$ & \multirow{2}{*}{0.71} \\
\hline Placebo group $(n=17)$ & $8(47)$ & 1.00 & \\
\hline \multicolumn{4}{|l|}{$35-35^{+6}$ weeks } \\
\hline Corticosteroid group $(n=64)$ & $18(28)$ & 1.32 (0.67 to 2.60$)$ & \multirow{2}{*}{0.41} \\
\hline Placebo group $(n=47)$ & $10(21)$ & 1.00 & \\
\hline \multicolumn{4}{|l|}{$\geq 36$ weeks } \\
\hline Corticosteroid group $(n=66)$ & $11(17)$ & $0.92(0.44$ to 1.93$)$ & \multirow{2}{*}{0.82} \\
\hline Placebo group $(n=66)$ & $12(18)$ & 1.00 & \\
\hline
\end{tabular}

While a systematic review of corticosteroid use in full term pregnancies suggests that further studies are required to investigate its effect on neonatal outcomes, ${ }^{21}$ there are safer and more effective measures of preventing respiratory disorders, such as avoiding unnecessary caesarean sections and delaying elective caesarean sections, whenever indicated, until after 39 weeks of pregnancy ${ }^{2223}$

Antenatal administration accelerates the effect of endogenous corticosteroids and induces the production of all the known components of the surfactant system. ${ }^{1324}$ As respiratory distress syndrome is characterised by a qualitative and quantitative deficiency of pulmonary surfactants, infants born before 34 weeks' gestation, in whom the incidence of this syndrome is higher, benefit from the administration of antenatal corticosteroids. ${ }^{145}$ Respiratory distress syndrome, however, is much less common in late preterm infants, in whom the principal respiratory disorder is transient tachypnoea. This develops from a delay in the absorption of lung fluid or in its inadequate clearance from the upper airways, resulting in more fluid in the lungs and reduced pulmonary complacency, leading to the onset

Table 5|Other complications and neonatal outcomes in infants born at 34-36 weeks according to antenatal treatment with corticosteroids or placebo. Figures are numbers (percentages) unless stated otherwise

\begin{tabular}{lcccc} 
Variable & $\begin{array}{c}\text { Corticoster- } \\
\text { oid }(\mathrm{n=143)}\end{array}$ & $\begin{array}{c}\text { Placebo } \\
(\mathrm{n}=130)\end{array}$ & $\begin{array}{c}\text { Risk ratio } \\
(95 \% \mathrm{Cl})\end{array}$ & $\mathrm{P}$ value \\
Jaundice & $76(53)$ & $73(57)$ & $0.94(0.76$ to 1.17$)$ & 0.57 \\
\hline Jaundice requiring phototherapy & $34(24)$ & $49(38)$ & $0.63^{*}(0.44$ to 0.91$)$ & 0.01 \\
\hline Hypoglycaemia & $15(11)$ & $9(7)$ & $1.50(0.68$ to 3.32) & 0.31 \\
\hline Neonatal sepsis & $6(4)$ & $9(7)$ & $0.60(0.22$ to 1.64$)$ & 0.32 \\
\hline Neonatal morbidity & $88(62)$ & $93(72)$ & $0.86(0.73$ to 1.02$)$ & 0.08 \\
\hline Admission to intensive care & $47(33)$ & $43(33)$ & $0.99(0.71$ to 1.39$)$ & 0.97 \\
\hline Neonatal death & 0 & $2(2)$ & $-\dagger$ & $0.23 \ddagger$ \\
\hline <4 days in hospital & $73(51)$ & $53(41)$ & $1.25(0.96$ to 1.63$)$ & 0.09 \\
\hline Mean (SD) days in hospital & $5.1(6.1)$ & $5.2(4.3)$ & - & 0.87 \\
\hline
\end{tabular}

*Number needed to treat=7 ( 4 to 33 ).

†Not estimated.

fFisher's exact test. of symptoms..$^{25}$ Antenatal corticosteroids could facilitate clearance of fetal lung fluid by activating the epithelial sodium channels and increasing ion transport, ${ }^{2026}$ though this mechanism has not been confirmed by other studies.

Another finding that merits debate concerns the reduction in the risk of neonatal jaundice requiring phototherapy, which we found was the only beneficial effect of corticosteroids. Other clinical trials had already shown this effect in premature infants born before 34 weeks' gestation, ${ }^{27}$ and it is possible that liver maturation could also be accelerated with corticosteroids. Nevertheless, the actual mechanisms underlying this effect and their implication in reducing neonatal morbidity in late preterm infants remain to be clarified.

\section{Limitations of study}

In our study, $43(13 \%)$ pregnant women were discharged after randomisation, went on to give birth in another hospital, and were lost to follow-up. Most of these women were discharged after 35 weeks of pregnancy and some could have reached full term; therefore, it is unlikely that significant rates of respiratory disorders or other neonatal morbidities could have been affected by the administration of corticosteroids. We believe that these losses, albeit a limitation of the study, had no effect on our conclusions.

Powering a study to detect a $50 \%$ reduction always runs the risk of missing smaller but clinically important reductions in the primary outcome. We do not believe that this has occurred because rates of respiratory morbidity, especially transient tachypnoea, were similar between groups. The real impact of steroids on the rates of respiratory distress syndrome remains to be established as these are quite low and our study was not powered to detect real differences between the groups.

\section{Conclusions and policy implications}

In the medical care of high risk pregnancies, the dominant concept, up until recently, suggested that there would be little need for further concern regarding neonatal outcomes after 34 weeks of pregnancy. Recent evidence has not supported this. Despite having low mortality rates, late preterm infants have been shown to be affected by clinically important morbidity, and a review of the evidence in support of current clinical practice is warranted. Unfortunately, our study suggests that antenatal treatment with corticosteroid does not result in significant reduction in neonatal respiratory morbidity.

Although the "safe" limit for interrupting a high risk pregnancy remains to be established, allowing the baby to remain in the uterus as long as maternal conditions and fetal wellbeing permit seems to be the key to preventing the complications of prematurity. We do not recommend delaying delivery in women with high risk pregnancies. What we are proposing, if we recognise the high neonatal morbidity in late preterm infants, is changing the paradigm of interruption of pregnancies reaching 34 weeks, based on the traditional belief that these infants are as physiologically and metabolically 


\section{WHAT IS ALREADY KNOWN ON THIS TOPIC}

Antenatal treatment with corticosteroids has been used to accelerate lung maturation in preterm infants, especially those born before 34 weeks' gestation

The effect of corticosteroids is mediated through acceleration of endogenous corticosteroids and increased production of all the components of the surfactant system

Preterm infants born between 34 and 36 weeks also have increased risk of respiratory disorders, mainly transient tachypnoea

\section{WHAT THIS STUDY ADDS}

The incidence of respiratory disorders (respiratory distress syndrome and transient tachypnoea) is not affected by antenatal administration of two doses of betamethasone after 34 weeks' gestation

Antenatal betamethasone given after 34 weeks' gestation reduced the risk of neonatal jaundice requiring phototherapy, possibly because of acceleration of liver maturation

mature as term newborns. According to current knowledge about this population, we believe that expectant management should be adopted whenever maternal conditions and fetal wellbeing allow in an attempt to prevent complications of prematurity.

Our results, albeit robust, could reflect the situation adopted in this protocol of a single centre and healthcare system. Additional randomised trials should be conducted to avoid hasty conclusions in the light of a single study. A large randomised multicentre clinical trial would be ideal to confirm these findings with a sufficient number of patients to detect small or occasional benefits of antenatal corticosteroids in cases of late prematurity. Furthermore, a meta-analysis of various future randomised clinical trials could encompass a larger number of patients and therefore strengthen and give greater relevance to our findings. The US National Institute of Child Health and Human Development (NICHD) is currently recruiting a target of 2800 women into its antenatal late preterm steroids (ALPS) trial, with the final analysis expected in 2013 (http://clinicaltrials.gov/ct2/show/NCT01222247).

Until these studies become available, current evidence does not justify the routine use of corticosteroids to prevent respiratory morbidity in late preterm infants. Incorporating these results into the available Cochrane review ${ }^{15}$ would help clinicians in deciding whether or not to prescribe steroids in women over 34 weeks' gestation.

Contributors: AMFP developed the initial protocol, initiated and coordinated the enrolment of patients, and drafted the manuscript. ICC reviewed and amended the study protocol, contributed to enrolment of patients, interpreting data, and writing the final version of the manuscript.JBC reviewed and amended the study protocol, and contributed to interpreting data and writing the final version of the manuscript.MMRA conceived the trial, developed its initial protocol, analysed the data,jointly drafted and reviewed the final version of the manuscript, and is guarantor.

Funding: This study was supported by the Instituto de Medicina Integral Prof Fernando Figueira-IMIP (www.imip.org.br), a private, not for profit healthcare organisation based in Recife, Pernambuco, Brazil, where the study was carried out. The institute did not interfere with study design of analysis and the funding covered all study expenses, including purchase of the drug and placebo.

Competing interests: All authors have completed the Unified Competing Interest form at www.icmje.org/coi_disclosure.pdf (available on request from the corresponding author) and declare: no support from any organisation for the submitted work; no financial relationships with any organisations that might have an interest in the submitted work in the previous three years; no other relationships or activities that could appea to have influenced the submitted work

Ethical approval: This study was approved by the Institutional Review Board (protocol 1029/2007). All the participants voluntarily agreed to take part in the trial and gave written informed consent.

Data sharing: Technical appendix, statistical code, and dataset available from the corresponding author at melamorim@uol.com.br. Participants gave informed consent for data sharing.

1 Young PC, Glasgow TS, Li X, Guest-Warnick G, Stoddard G. Mortality of late-preterm (near-term) newborns in Utah. Pediatrics 2007;119:e659-65.

2 Kramer MS, Demissie K, Yang H, Platt RW, Sauvé R, Liston R. The contribution of mild and moderate preterm birth to infant mortality. Fetal and Infant Health Study Group of the Canadian Perinatal Surveillance System. JAMA 2000;284:843-9.

3 Wang ML, Dorer DJ, Fleming MP, Catlin EA. Clinical outcomes of near term infants. Pediatrics 2004;114:372-6.

4 Tomashek KM, Shapiro-Mendoza CK, Weiss I, Kotelchuck M, Barfield W, Evans S, et al. Early discharge among late preterm and term newborns and risk of neonatal morbidity. Semin Perinatol 2006;30:61-8.

5 Davidoff MJ, Dias T, Damus K, Russell R, Bettegowda VR, Dolan S, et al. Changes in the gestational age distribution among US singleton births: impact on rates of late preterm birth, 1992 to 2002. Semin Perinatol 2006;30:8-15.

6 Santos IS, Matijasevich A, Domingues MR, Barros AJ, Victora CG, Barros FC. Late preterm birth is a risk factor for growth faltering in early childhood: a cohort study. BMC Pediatr 2009;9:71.

7 Fuchs K, Wapner R. Elective cesarean section and induction and their impact on late preterm births. Clin Perinatol 2006;33:793-801.

8 Bird TM, Bronstein JM, Hall RW, Lowery CL, Nugent R, Mays GP. Late preterm infants: birth outcomes and health care utilization in the firs year. Pediatrics 2010;126:e311-9.

9 Escobar GJ, Clark RH, Greene JD. Short-term outcomes of infants born at 35 and 36 weeks' gestation: we need to ask more questions. Semin Perinatol 2006;30:28-33.

10 Jain L, Eaton DC. Physiology of fetal lung fluid clearance and the effect of labor. Semin Perinatol 2006;30:34-43.

11 Jain L. Respiratory morbidity in late preterm infants: prevention is better than cure! Am J Perinatol 2008;25:75-8.

12 Ventolini G, Neiger R, Mathews L, Adragna N, Belcastro M. Incidence of respiratory disorders in neonates born between 34 and 36 weeks of gestation following exposure to antenatal corticosteroids between 24 and 34 weeks of gestation. Am J Perinatol 2008;25:79-83.

13 Bonanno C, Wapner RJ. Antenatal corticosteroid treatment: what's happened since Drs Liggins and Howie? Am J Obstet Gynecol 2009;200:448-57.

14 Crowley PA. Antenatal corticosteroid therapy: a meta-analysis of the randomized trials, 1972 to 1994. Am J Obstet Gynecol 1995; $173: 322-35$

15 Roberts D, Dalziel S. Antenatal corticosteroids for accelerating fetal lung maturation for women at risk of preterm birth. Cochrane Database Syst Rev 2006;3:CD004454.

16 Liggins GC, Howie RN. A controlled trial of antepartum glucocorticoid treatment for prevention of the respiratory distress syndrome in premature infants. Pediatrics 1972;50:515-25.

17 Amorim MM, Santos LC, Faúndes A. Corticosteroid therapy for prevention of respiratory distress syndrome in severe preeclampsia. Am J Obstet Gynecol 1999;180:1283-8.

18 Kurl S, Heinonen KM, Kiekara O. The first chest radiograph in neonates exhibiting respiratory distress at birth. Clin Pediatr 1997;36:285-9.

19 Sinclair JC. Meta-analysis of randomized controlled trials of antenata corticosteroid for the prevention of respiratory distress syndrome: discussion. Am J Obstet Gynecol 1995;173:335-44.

20 Stutchfield P, Whitaker R, Russell I, Antenatal Steroids for Term Elective Caesarean Section (ASTECS) Research Team. Antenatal betamethasone and incidence of neonatal respiratory distress after elective caesarean section: pragmatic randomised trial. BMJ 2005;331:662.

21 Sotiriadis A, Makrydimas G, Papatheodorou S, loannidis JP. Corticosteroids for preventing neonatal respiratory morbidity after elective caesarean section at term. Cochrane Database Syst Rev 2009;4:CD006614.

22 Zanardo V, Simbi AK, Franzoi M, Soldà G, Salvadori A, Trevisanuto D Neonatal respiratory morbidity risk and mode of delivery at term: influence of timing of elective caesarean delivery. Acta Paediatr 2004;93:643-7. 
23 Riskin A, Abend-Weinger M, Riskin-Mashiah S, Kugelman A, Bader D. Cesarean section, gestational age, and transient tachypnea of the newborn: timing is the key. Am J Perinatol 2005;22:377-82.

24 Ballard PL, Ballard RA. Scientific basis and therapeutic regimens for use of antenatal glucocorticoids. Am J Obstet Gynecol 1995;173:254-62.

25 Guglani L, Lakshminrusimha S, Ryan RM. Transient tachypnea of the newborn. Pediatr Rev 2008;29:e59-65.
26 Helve O, Pitkänen O, Janér C, Andersson S. Pulmonary fluid balance in the human newborn infant. Neonatology 2009;95:347-52.

27 Gamsu HR, Mullinger BM, Donnai P, Dash CH. Antenatal administration of betamethasone to prevent respiratory distress syndrome in preterm infants: report of a UK multicentre trial. $\mathrm{Br}$ J Obstet Gynaecol 1989;96:401-10.

Accepted: 30 January 2011 